\title{
Index
}

Note: 'n.' after a page reference indicates the number of a note on that page.

Aap Kaa Hak 218 see also Pande, S.

Abel-Smith, B. 42, 46

accent $8,125,135-6,164$,

223,275

see also language skills

activist doctors $51,211-38,252$

see also agency

agency $150,153,174$

see also activist doctors

Ahuja, R. 157-8, 260

Ahuja, S. K. 157-8, 246-7, 260

alcohol

consumed by doctors 101 ,

125,136

dependency 7

Alma Ata conference 47

see also World Health

Organization (WHO)

alumni organisations 236-8

America 4, 8, 38-9, 112-13, 285

see also USA

Andeanism 279

see also migration, history of

anglicised doctors $65,84 \mathrm{n} .28,111$,

$135,220-3$ apartheid

medical 130

in South Africa 152, 221-2

see also Lawrence, R. A. A. R.

Appalachian 8

Australia 4, 7, 95

doctors from 36, 151

emigration to $38-9,43$

see also Collings, J.

Balint, M. 48, 269

see also biopsychosocial model of general practice; European doctors; refugee doctors

Banatvala, E. \& J. 193-4

Bangladesh 100, 155, 186 doctors from 1-2, 19n.1, 99, 130, 226-7, 239n.14, 286

Bangladesh Medical Association 226

Battersea 65, 215

see also Gulati, H.L.

Bevan, A. 2, 33, 70, 170, 174

see also NHS, establishment of;

Wales; Labour party

Bhanumathi, K. S. 117-18, 120n.5, $140,156,243 n .117$ 
biopsychosocial model of general practice 48, 262, 269-70 see also Balint, M.; general practice Birmingham 65-7, 142, 151, 159-60, $168,171,173,194,200,214$, 227, 232, 234 see also Prem, D.; Venugopal, S.

Bishop Cotton School 116

Blyton, E. 134-5

BMA see British Medical Association

Bolton 66, 225, 248-9, 259 see also Manchester

Boomla, F. 63-4, 67 see also Boomla, R.; Kutar, S.; Parsees; Zoroastrian

Boomla, R. 63-4, 67, 116, 216, 239n.35 see also Boomla, F.; Kutar, S.; Parsees; Zoroastrian

British Medical Association (BMA) Conference speaker's views on migrant doctors 81

Empire Advisory Bureau 70 enquiries to made by overseas doctors 79, 169

International Medical Visitors Bureau 70 meetings with DHSS and GMC about overseas doctors 165-6 migrant doctors prominent in the 219-21, 231

official's views of migrant doctors 74 plans to reduce numbers of migrant doctors 224 see also General Medical Services Committee (GMSC); Husain, M.H.; Lawrence, R. A. A. R.

British medical graduates exodus/emigration of $39,42-5$, 71,283 preference given to 129-31, 143-4

see also migration, medical; white doctors

Byrne, Prof. P. 265 see also Manchester, University of

Canada medical migration to $7,8,38,39$, 43-4, 95

cardiology 74, 154, 235, 259-60 see also heart disease

Castle, B. 227, 251 see also Labour Party

Ceylon 63, 97, 99 see also Chandran, R.; Sri Lanka

Chandran, R. 99, 115, 117, 119, 207n.27, 213-14 see also Ceylon; Conservative Medical Society; Conservative Party; Sri Lanka; Vaughan, G.

Chatterjee, S. 104, 116-17 see also Overseas Doctors' Association (ODA)

Chaudhuri, A. 121n.28, 122n.66, 216-17, 223, 243n.120, 257, 276n. 16 children doctors' experiences as 97, 111-14, 134

Chowdary, D. S. \& S. 65, 185 'civis Britannicus sum' 114 see also Empire class

attitudes linked to $41,72-3$, 118-19, 141-2, 203-4 see also middle class; working-class areas 
coalfield communities 52, 167, 182, 186-7, 213-14, 282

see also industrial areas; working-class areas

Collings, J. 36, 51

colonialism see Empire

Commission for Racial Equality commissioners of the 223

report by the 77-8, 142 see also Chandran, R.; Ray, D.

Committee of Inquiry into the Regulation of the Medical

Profession 71, 81

Community Relations

Commission 224

Conservative Medical Society

217,223

see also Chandran, R.; Conservative

Party; Vaughan, G.

Conservative Party 33-4, 74, 76,

214-17, 228

see also Chandran, R.,

Conservative Medical Society;

Gulati, H.L; Powell, E.;

Thatcher M.; Vaughan, G.

Coventry 183, 214

cricket see Test match

Crossman, R. 82

cultural transfer 215, 247, 255,

269-70, 282

Department of Health and Social

Security (DHSS) 218

depression 219, 269

in doctors 107

Derbyshire 187, 219, 269

see also Lawrence, R. A. A. R.

Dhaka 98-9, 108, 256 see also Bangladesh

DHSS see Department of Health and

Social Security

diabetes 49, 196, 219, 234, 259-62

diaspora, South Asian medical

$$
\text { 63-6, 108-18 }
$$

difference 130-40

see also heterophobia

'dirty work' 17, 72, 156, 182, 191

discrimination

in general practice 124-62, 250, $258,270,274-5$

in medicine 6, 67-75, 130, 229

see also difference;

heterophobia; racism

discussions with GMC and BMA

regarding migrant doctors 165-6

relationship with Overseas

Doctors' Association (ODA)

229-30, 235

distribution

of doctors 40-1, 67-8, 282

of GPs 55n.39, 160, 170-4

see also discrimination; NHS staff

shortages

diversity of the medical profession $202,283-4$

see also cultural transfer; religion

Doctor in the House 104

'doctors for miners' 216

doctor shortages see distribution,

of doctors/GPs; NHS, staff

shortages in

Donaldson, Sir L. 228-9

drug companies 238

ECFMG see Educational Council for Foreign Medical Graduates 
see also USA

Educational Council for Foreign

Medical Graduates

(ECFMG) 95

'Ellis islands' 3, 285

see also 'imagined communities';

Parekh Report

emigration of British doctors see

British medical graduates, exodus/emigration of

Empire

legacies of and British healthcare 8-9, 60-90, 220-5

and medicine in colonial

India 61-3

in/of the mind 93-123

positive feelings towards

109-13

resistance to British 112, 216

England ix-x, 1, 33, 36-40, 42,

55n.39, 62-3, 65-6, 71, 97,

99-100, 102-4, 106-9, 111,

$113-19,135,137,143,145$,

164-5, 168-9, 171-2, 184,

186, 194, 200, 207n.17, 214,

218-19, 225, 232, 234-6, 248,

$255,260,282$

Ennals, D. 227-8

Esmail, A.

arrested by police and pursued by

GMC 147n.24

research on racism in

medicine $6,81,82 \mathrm{n} .1$,

129, 171-2

Essex 37-8, 65, 142, 168, 173, 185

ethnicity 125, 129-31, 134, 136,

$139,159,183,218-23,253$,

273,284 see also heterophobia;

patients; racism

European doctors 133, 161, 163-4, 168, 173

see also Balint, M.; refugee doctors

Executive Councils 161, 168, 173, 248

see also Family Practitioner

Committee (FPC)

Family Doctors' Charter 39, 49, 168,261

Family Practitioner Committee (FPC) 173, 199-200, 248, 272-3

Fanon, F. 5, 94

Fawley, Lord Hunt of 230

Fellowship of the Royal College of Physicians (FRCP) 275

Fellowship of the Royal College of Surgeons (FRCS) 100,268

female doctors $17,84 \mathrm{n} .28,133$, 139-40, 163-4, 198-201 discrimination against 133 , 139-45, 159, 205, 273-4 proportion in migrant doctor population 71

Ferris, P. 141-2, 174, 189

Fife 186, 194

see also Scotland

FPC see Family Practitioner

Committee

FRCP see Fellowship of the Royal

College of Physicians

FRCS see Fellowship of the Royal

College of Surgeons

gender see female doctors;

heterophobia; women('s) 
General Medical Council (GMC) 9, $39,43,82,105,113-14,165-6$, 230, 286

hearing concerning a South Asian doctor 183 overseas inspection teams

$81,106-7$ recognition of South Asian

degrees by $62,75,80,95-6,226$ representation of South Asian

doctors on 229, 235, 274 see also Esmail, A.; immigration policy, British; Medical Act (1978); Professional and Linguistic Assessment Board (PLAB); Temporary

Registration Assessment Board (TRAB)

General Medical Services Committee (GMSC) 37, 40, 173

South Asian doctors sitting on the $127,221,229,231$ see also Husain, M.H.; Overseas

Doctors' Association (ODA); Ray, D.

general practice 'cornerstone' of the NHS 1 , $31-59,244$ entry into $43,65,124-70$ as a medical specialty $10,45,48$, $53,232,244,261-2$ as a mosaic $262-78$ as a 'post-modern' specialty 53 , 268, 273 'renaissance' of 45, 48-50, $53,244-78$ see also biopsychosocial model of general practice; discrimination; distribution, of doctors/GPs; patients; practice premises; primary care; racism

geriatrics 6, 74-5, 132, 157, 174

Germany

doctors from 164-5

migrant doctors in 23, 99

Ghalib, M. A. K. 102

Gilani, S. A. A. 106-7, 111-12, 136-7, 175n.12, 230, 232, 236, 264-7

see also Overseas Doctors' Association (ODA)

Gish, O. 26, 77-8

Glasgow x, 52, 164, 194 see also Kausar, M.S.; Scotland GMC see General Medical Council

GMSC see General Medical Services Committee

GP cooperatives 225 see also Korlipara, K.

GP training see postgraduate medical training

Grey-Turner, E. 74

Gulati, A. 67

Gulati, H. L. 65, 214-15

Gypsies, Roma and Travellers see Patients, Gypsy, Roma and Traveller

'hands-on' doctors 253-62 see also medical cultures Hart, J. T. 49-51, 271, 282 see also inverse care law; Wales; working-class areas

health centres $47,49,65,206$, $246,248-53$ 
health visitors 49,253

heart disease

in doctors $162-3$

in patients $8,192,196,219$,

234, 259-61

see also cardiology

heterophobia 17, 125-46, 150, 158, $162,174,184,187-9,206,233$, 270,276

see also difference; Islamophobia;

racism

Heysham 248

see also Morecambe

High Wycombe 199-200

Hindi 146, 218, 277n.46

see also Aap Kaa Hak; Urdu

Hindus 106, 116-17, 123n.79, 128, 219, 286

History and Policy 11-12, 279-88

HIV/AIDS see patients, with HIV/ AIDS

Home Office

manipulation of immigration statistics by $14,79-80$

policies on recruitment of overseas-trained doctors 69 see also immigration policy, British

Horder, J. 47 and 'renaissance' of general practice 50

hospital medicine 2, 7, 10, 34-6, 42, $45-7,49,70-3,78,80-1,97$, $107-8,120,124-6,136,142-3$, $155-6,169,184-5,205,219$, $232,235,237,248,253-9,261$, 263, 266, 268

junior posts in 6, 43, 70-1, 74, $78-9,156$ perceptions of migrant doctors in 67-9, 127-32, 135, 137-9, $152,187-8$

humour 192, 281

see also laughter

Husain, M. H. 221, 223-4

industrial areas $1,6,7,31,36,40-1$, $49,51-2,66-7,70,74,133$, $145,150,162,165,167-8$, 170-4, 194

see also coalfield communities;

peripheral areas;

working-class areas

'imagined communities' 284-6

see also 'Ellis Islands'; Parekh report immigration debates $12,284-7$ immigration policy, British 9, 75-82, 284-7 see also General Medical Council (GMC); Home Office; patrial connection; voucher scheme/ system

immunisation 256

see also public health; preventive medicine; vaccination India(n) 47, 61-3, 82n.1, 93, 97, 99, 109-10, 112-16, 204-5, 216, 226-7, 234, 236-7, 246, 255-6 doctors from 1, 5, 7-8, 19, 25n.59, $47,61-8,70-2,93,96-7,100-1$, $124,130,139,141,146,162$, $168-70,172-3,183,188,204$, 226, 231, 240n.48, 252, 286 migrants from 159, 161, 197 partition and independence of 9, 119 see also Empire 
Indian Medical Association

(Great Britain) 69, 226

Indian Medical Society 226

'International' Health Service 1-3

International Medical Graduates

8-9, 44, 95, 124-5, 286

see also migration, medical

inverse care law 282

see also Hart, J. T.

Ireland

doctors from 42, 69, 133, 163,

168-9, 173

Northern 25n.65, 163, 168

Irvine, Sir D. 39, 73-4

Islamophobia 205

see also Muslims

Izzet 96

see also Empire, in/of the mind

Jenkin, P. 228

Jewish doctors 145, 164-5

see also European doctors; refugee doctors

Joshi, H. 144-5, 247, 207n.23, 254-5, 264, 272

Kamal, L. R. M. 99, 121n.26, 121n.31, 122n.64, 207n.24, 212-13, 215-16, 252-3, 277n.20, 277n.41

Kannada Balaga 237

Kapur, H. L. 105, 113-14, 137-8, 164, 248

Kataria, M. S. 226 see also Indian Medical Association (Great Britain)

Kausar, M. S. 154, 156, 209n.56, 275-6, 278n.47

Khaled, M. A. 175n.5, 186-7
Kinnock, N. 216 see also Labour Party; Ray, D.

Kipling, R. 233

Korlipara, K. 148n.50, 207n.6, 225, 229, 239n.22, 242n.105, 248-9, 274, 277n.29, 277n.37

see also GP Cooperatives; Overseas

Doctors' Association (ODA)

Kotwall, F. B. 116, 119, 165, 207n.28

Kureishi, H. 281

Kutar, S. 64, 195, 204, 256 see also Boomla, F.; Boomla, R.;

Parsees; Zoroastrian

Labour Party 33, 76, 82, 214-17, 227, 251

see also Bevan, A.; Castle, B.;

Kinnock, N.; Gulati, H. L.;

Prem, D.; Ray, D.

language skills $81-2,134-5$, 198-9, 202-3

see also accent

laughter 110-12, 136-7, 265, 275

see also humour

Lawrence, R. A. A. R. 112, 152-3, 187, 191, 219, 221-3, 240n.46

Leicester 13, 73, 161, 197, 218, 227 see also Sayeed, A.

Liberal Party 214, 226 see also Prem D.; Smith, C.

limited registration 80,230

Liverpool 162-3, 188, 218, 272 see also Pande, $S$.

Local Medical Committee (LMC) $145,196,249-50,275$

London 40, 47, 64-7, 70, 76, 99, 102, 106, 113, 129, 137-8, 141, $143,171,188,193-5,199,202$, $215,237,273,287$ 
Borough of Camden 163-4, 199

County Council 215

Greater 65, 170-3

Majumdar, R. K. 41, 110, 164, 208n.32, 272-3, 277n.45

Mallick, Sir N. 67, 209n.76

management of GP practices 271-2

Manchester 6, 66, 132, 160, 170-1, 191, 194, 197, 248, 251, 272-3, 275

University of 264-5

Mather, H. 262

see also diabetes

Medical Act (1978) 80

see also General Medical

Council (GMC)

medical auxiliaries 283

medical cultures 52, 75, 99, 104, 108, $117-18,120,131,138,144$, $211,233,255,281-4$

see also cultural transfer; 'handson' doctors; medical training

Medical Defence Union 258 'medical dependency' 61-3, 98-102

see also Empire, of/in the mind; Izzet

medical frontier zone 181

see also coalfield communities; distribution, of doctors/GPs; industrial areas; peripheral areas; working-class areas medical politics $48,50,52,128,211$, 219-31, 238, 245, 251

Medical Practitioners' Union (MPU) 168, 221

see also Ray, D. medical regulations see Committee of Enquiry into the Regulation of the Medical Profession; General Medical Council (GMC); Medical Act (1978); Professional and Linguistic Assessment Board (PLAB); temporary registration; Temporary Registration Assessment Board (TRAB)

medical schools

associations see alumni organisations

in Amritsar 106

in Birmingham 232, 234

British 42, 44, 67, 69, 71, 127, $142-3,168,216,226,228,283$ overseas 81

in Malawi 52

South Asian 61-2, 80, 94-108, 233, 236

St George's 129 medical specialties unpopular 69, 72-4, 126 see also distribution, of doctors /

GPs; general practice as a medical specialty; geriatrics; psychiatry; royal colleges medical training $17,42,70,78,104$, $138,216,283$

as a form of 'aid' 78

postgraduate $38,77-9,99-10$, $120,130,144,230,232-6,250$, 268, 272

in South Asia 94, 97

in the Soviet Union 96

see also cultural transfer; medical cultures

medicine as an art 255, 269 
Medicos journal 233-4

Membership of the Royal College of General Practitioners (MRCGP) 89n.143, 232, 234, 262, 268, 275

Membership of the Royal College of Physicians (MRCP) 89n.143, $100,268,275$

Membership of the Royal College of Surgeons (MRCS) 275

Merrison Committee see Committee of Enquiry into the Regulation of the Medical Profession

middle class $41,67,72-3,97$, 109-10, 115-20, 142-3, 145, $183,188,202-3,217,280$

Middle-Eastern countries 234 doctors 133, 151, 173

migrant doctors see migration, medical migration history of $\mathrm{ix}-\mathrm{x}, 3-5$, 279-80, 284-7 and the making of Britain xiii, $5,281-7$ medical 3-9, 14, 16, 18, 31-2, $60-1,77,79$ see also International Medical Graduates mining 1, 38-9, 67, 146, 174, 191, $212-13,215,252$ see also coalfield communities; miners; miners' strike miners $67,187,213$ miners' strike 216 Ministry of Health see Department of Health and Social Security (DHSS)

'missing the revolution' 279 see also migration, history of
Mohan, P. R. L. 97, 113, 132, 203

Morecambe 164 see also Heysham

MRCGP see Membership of the Royal College of General Practitioners MRCP see Membership of the Royal College of Physicians

MRCS see Membership of the Royal College of Surgeons

Muslims 4, 116-17, 128, 136, 139-40, 198, 205, 215, 218, 286

Muthu, C. 68

New Zealand(er) 4, 134 doctors emigrating to 39,43 doctors from 8

NHS

establishment of 32-5, 61, 174, 194 funding difficulties of 33-4, 45-8, 69,247

(rethinking the) history of 34, 280-1

migrant domestic and catering staff in the 72 opposition to $9-10,34,37-8$, $42,44-5$ as a postcolonial institution $69,82 \mathrm{n} .1$ staff shortages in 69-70, 282-3, 285-7

Nigeria, doctors from 234 nurses 49, 69, 147n.9, 205, 248, 253, 272, 283, 286-7

Black and Minority Ethnic 132-3, 205

migrant 3, 69, 72, 132, 205

ODA see Overseas Doctors'

Association 
on call $41,206,260$

see also out of hours provision open areas in general practice $158-60$

oral history 2, 6, 10-18, 34, 63, $101,153,159,164,182$, 184, 193, 203-4, 250, 252,261

out of hours provision 41 ,

191-2, 225

see also GP cooperatives; on call

Overseas Doctors' Association (ODA)

73, 116, 226-38, 262, 274

collaboration with DHSS

229-30, 235

establishment of 19, 73, 224

Oxfam report on medical

migrants 283

'pairs of hands' 73, 104, 124,

150,232

Pakistan(i) 72, 76, 93-4, 97, 99, 106-7, 115, 157, 161, 197-8, 209, 234

doctors from 1, 19n.1, 70-1, 93, $130,139,168-73,186,188$, 205, 286

Pakistani Welfare and Information Centre 197

Pakistan Medical Graduates

Association 226

Pakistan Medical Society 226, 236

Pande, S. 162-3, 218, 228 see also Aap Kaa Hak; Liverpool

Parekh report 284

Parsees 64, 165, 202

see also Boomla, F.; Boomla, R.; Kutar, S.; Zoroastrian
Partition see India, partition and independence of

Pathak, P. L. 103, 160, 175n.14, 198, 201, 207n.30, 264

patients $34,37,41,44-6,50-2,75$, 81, 82n.2, 103-4, 127, 131, 141-2, 159, 163-4, 168, 170, $173,181,183-4,189,191-2$, $212,217,221,225,247-8,254$, 256, 263-4, 269, 273-4, 284 doctors' relationship with1-2, 16, $19,139,194-206$ ethnic minority $187,194-205$, 229-30, 269-70

female 198-200

Gypsy, Roma and Traveller 195-6, 204

lists 49, 261 middle class 67 older 6, 75, 190-1, 214, 218 South Asian 218-19, 269-70, 273, 281-3 with HIV/AIDS 202, 284 white $187-8,202$ working-class 41, 162, 203, 205-6, 212-13, 220, 273 patrial connection to Britain 77 see also immigration policy, British; voucher scheme/ system patronage 126, 167 pay see remuneration of doctors peripheral areas 55n.39, 73, 181, 187,192 see also coalfield communities; inner-city areas; working-class areas

PLAB see Professional and Linguistic Assessment Board 
postgraduate medical training see Fellowship of the Royal College of Physicians (FRCP); Fellowship of the Royal College of Surgeons (FRCS); general practice, 'renaissance' of; medical training, postgraduate; Membership of the Royal College of General Practitioners (MRCGP); Membership of the Royal College of Physicians (MRCP); Membership of the Royal College of Surgeons (MRCS)

Powell, E. 74, 76, 78, 216 'rivers of blood' speech 76, 78 South Asians and the views of 203-4

see also Conservative Party practice premises 52, 163, 246-53, $256,259,261,273$

Prasad, R. 100-2, 152, 154, 259

Prem, D. 65, 214, 224 see also Sayeed, A.; Standing

Conference of Asian

Organisations

preventive medicine $36,46-7$, $49,260-1$

see also immunisation; public health; vaccination primary care $40,46-50,67,124-5$, 150, 163, 167-70, 172-4, 199, 244, 251-3, 256, 258-61 see also Alma Ata conference; general practice professional development 231-8 see also general practice, as a medical specialty; medical training, postgraduate

Professional and Linguistic Assessment Board (PLAB) 80, 89n.143

see also Temporary Registration

Assessment Board (TRAB)

psychiatry $140,164,174,184$

public health $51,65,215,219,283$

see also immunisation; preventive medicine; vaccination

Qureshi, S. M. 233-4

racism $75,184,218$

anti- 182, 196, 223

in British society 182, 185

in medicine 6, 125-46, 158, 162, 174, 183-4, 233, 270, 272, 276,280

of a number of South Asian doctors 205-6, 283

see also difference; heterophobia; Islamophobia

Raj see Empires

Rao, R. N. 99, 104-5, 110, 276n.8

Rao, U. 112-13, 156, 207n.20

Ray, D. 116, 127-9, 156, 195-6, 192, 213, 216, 221, 223-4, 238n.3, 239n.17, 239n.19, 251-2

RCGP see Royal College of General Practitioners

refugee doctors 7,70

see also Balint, Michael; European doctors

religion $117,125,165,286$ 
see also alcohol; Hindus;

Islamophobia; Muslims; Sikh;

Zoroastrian

remuneration of doctors 90, 261

rickets see Stop Rickets campaign royal colleges $10,48,73,99$,

103,250

see also Fellowship of the

Royal College of Physicians

(FRCP); Fellowship of the

Royal College of Surgeons

(FRCS); medical training, postgraduate; Membership

of the Royal College of

General Practitioners

(MRCGP); Membership of

the Royal College of Physicians

(MRCP); Membership of the

Royal College of Surgeons

(MRCS); Royal College of

General Practitioners; Royal

College of Obstetricians and

Gynaecologists; Royal College

of Physicians; Royal College of

Surgeons

Royal College of General

Practitioners (RCGP) 39-40,

47, 50, 144, 172, 244, 258,

263-4, 270-6

see also general practice;

primary care

Royal College of Obstetricians and

Gynaecologists 101, 128, 151

Royal College of Physicians 35, 101

Royal College of Surgeons 63, 101,

106, 136

rugby $142-3$ see also sport; Test match

Ruxton, B. 65, 67

Salam, M. A. 1-2, 121n.32, 271-2, 278n.49

Saudi Arabia

South Asian doctors in 7

Sayeed, A. 73, 161, 163, 218, 224, 226-7, 230

see also Leicester; Overseas

Doctors' Association (ODA);

Prem D.

Scotland 33, 36, 40, 55n.39, 108, 186,235

doctors from 133

see also Fife; Glasgow

Sellars, P. 220, 223, 240n.48

Sen, A. K. 38-9, 118, 123n.67, 252

SEN see nurse, State Enrolled

sexism see discrimination; female

doctors; heterophobia; women('s)

Shah, N. R. 175n.1, 275n.58

shortages of doctors see distribution, of doctors/GPs; NHS, staff shortages in

Sikh 106

single-handed practices $37,49,51$, $158,162-3,165-6,186,191$, 206, 250

see also small practices

small practices $49,51,58 \mathrm{n} .93$

see also single-handed practices

Smith, C. 226

see also Liberal Party

Smith, D. J. 58n.93, 97, 101, 126-7, $129,133,172$ 
South Africa(n) 112, 152, 221 see also apartheid

Soviet Union, recognition of degrees from 96

see also Alma Ata conference; USSR

'special' labour 31, 33, 166

Spennymoor 165

sport 135, 142

see also rugby; Test match

Sri Lanka 62, 80, 93, 97, 99, 115 doctors from 1, 19n.1, 169-70, 172, 226, 286 see also Ceylon; Chandran, R. SRN see State Registered Nurse staff shortages see NHS, staff shortages in

Standing Conference of Asian

Organisations 224 see also Prem D.; Sayeed, A.

State Registered Nurse (SRN) 133 statistics on South Asian doctors in Britain 14, 63, 170-2

Stevenson, D. 82, 229

Stop Rickets campaign 230

Swani, M.S. 232

Tagore, R. 113

Talukdar, M. N. I. 109, 122n.47, 175n.7, 193, 206, 218, 232-3, 276n.11

temporary registration 80,230 see also General Medical Council

(GMC); Professional and

Linguistic Assessment Board (PLAB); Temporary

Registration Assessment Board (TRAB)
Temporary Registration Assessment Board (TRAB) 80 see also General Medical Council (GMC); Professional and Linguistic Assessment Board (PLAB); temporary registration

Test match 127, 147n.15 see also sport

Thatcher, M. positions on the NHS 10, 34 views on immigration 216 see also Chandran, R.;

Conservative Medical Society;

Conservative Party; Powell, E.

TRAB see Temporary Registration Assessment

Trades Union Congress (TUC) 223-4 see also Medical Practitioners' Union; Ray, D.

Urdu 111, 198-200, 218 see also Aap Kaa Hak; Hindi USA 4, 39, 95, 157 emigration to 39,43 migrant doctors in 7, 8, 44, 95, 164 see also America; Appalachian;

ECFMG; Verghese, A.

\section{USSR 47}

see also Soviet Union

vaccination 260

see also immunisation; preventive medicine; public health

vaughan, G. 223, 230

see also Chandran, R.;

Conservative Party,

Conservative Medical Society 
Valleys see Welsh Valleys

Venugopal, S. 118, 159, 160, 162, 200, 208n.44, 227, 229, 243n.114, 278n.48

see also Birmingham; Overseas

Doctors' Association (ODA)

Verghese, A. 7-8

vocational training for GPs see

general practice, as a medical

specialty; medical training,

postgraduate

voucher scheme/system 77, 79-80

see also Home Office; immigration

policy, British

von Kuenssberg, E. 40

Wales 33, 40, 42, 55n.39, 71, 144, 169, 171-2, 207n.17, 235, 251-2, 282

South 1, 41, 49, 52, 194-5, 247, $251,272-3$

see also Bevan, A.; coalfield communities; Hart, J.T.;

industrial areas; Joshi, H.; miners; miners' strike; mining; Ray, D.; Welsh Valleys

Watkins, S. 147n.20, 231

Welsh Valleys 41, 156, 164, 191, 195-6, 213, 272 see also Bevan, A.; coalfield communities; Hart, J.T.; industrial areas; Joshi, $\mathrm{H}$; miners; miners' strike; mining; Ray, D.; Wales

white doctors $1,8,127-9,132,138-$ 9, 144-6, 153, 163, 166, 182, $184,186,196,215,233,258$, $269,272-4$

see also British medical graduates women('s) 101, 133, 142, 164, 185-6, 197-9, 201, $230,269-70$ doctors 66, 71, 103-4, 131, 139, 144,153

history 11

see also female doctors; patients, female

working-class areas 41, 135, 162 see also coalfield communities; industrial areas; peripheral areas

World Health Organization (WHO) 47, 95

Yellowlees, H. 40, 226

Zoroastrian 202 see also Parsees; religion 
Julian M. Simpson - 9781526115782

Downloaded from manchesterhive.com at $04 / 26 / 2023$ 12:46:41PM 